\title{
Determinants of Nutrition Knowledge, Attitude and Practices of Adolescent Sports Trainee: A Cross- sectional Study in Bangladesh
}

\section{Md. Bakhtiar}

Department of Exercise Physiology, BKSP, Bangladesh

\section{Masud-ur Rahman}

Protibondhi Sheba o Sahajjo Kendro Magura, Ministry of Social Welfare

Md Kamruzzaman

Dept. of Applied Nutrition and Food Technology, Islamic University, Kushtia

\section{Nargis Sultana}

250 Bedded General Hospital, Meherpur

\section{Kh. Shafiur Rahaman}

Good Neighbors Bangladesh, Dhaka

Shaikh Shahinur Rahman ( $\nabla$ shahinanft@gmail.com )

\section{Research article}

Keywords: Nutrition, Knowledge, Attitude, Practices, Athletes, Sports

Posted Date: April 21st, 2020

DOl: https://doi.org/10.21203/rs.3.rs-19204/v1

License: (c) (i) This work is licensed under a Creative Commons Attribution 4.0 International License. Read Full License

Version of Record: A version of this preprint was published at Heliyon on April 1st, 2021. See the published version at https://doi.org/10.1016/j.heliyon.2021.e06637. 


\section{Abstract \\ Background}

Nutrition is an important predictor of an athlete's performance. It is more important for particularly those athletes who are in the growing stage (adolescents). This study aimed to assess their current level of knowledge, attitude, and practices (KAP) of nutrition and to predict potential factor impact on the level of these KAP.

\section{Methods}

A cross-sectional study was conducted among adolescent trainee athletes selected conveniently from 11 sports departments (e.g. cricket, football, hockey etc.) of Bangladesh Krira Shikkha Protishtan (BKSP). A semi-structured interviewer-administered questionnaire was used for data collection. Anthropometric measurements (e.g. height, weight, BMI, BF \%) were taken using appropriate methods. The data were analyzed using R (v3.6.1) and Python Jupyter Notebook. Descriptive statistics, t-test, analysis of variance (ANOVA) and logistic regression were used to determine the relationship between dependent and independent variables.

\section{Results}

A total number of 260 participants were enrolled in this study. Among them, $86 \%$ were male. Their mean age was $15.50 \pm 1.83$ ranging from 12-19 years. More than half of the participants had good nutrition knowledge $(n=149,57.3 \%)$. Fifty-six percent participants had negative attitude $(n=146)$ and $42.3 \%(n=$ $110)$ had poor level of practices regarding nutrition. Age $(p=0.007)$, gender $(p=0.004)$ and duration of sports training $(p=0.004)$ of participants were significantly associated with knowledge, while only age and BMI were significantly associated with practices. Athletes with less body fat were more likely to have good nutrition practice behavior (AOR $0.895 ; 95 \% \mathrm{Cl} 0.83,0.96 ; \mathrm{p}<0.05$ ) and nutrition knowledge was positively associated with nutrition practice (AOR $2.335 ; 95 \% \mathrm{Cl} 1.405,3.88 ; \mathrm{p}=0.001)$.

\section{Conclusion}

Insufficient knowledge, negative attitude, and poor practices were observed among many of our participants. Previous nutrition training was found as potential factor of good nutrition knowledge and practice. It is necessary to provide appropriate nutrition information to the adolescent athletes through proper educational intervention on a regular basis.

\section{Background}


Nutritional status is one of the determinants of physical fitness and training of a sports person [1]. Physical performance is influenced by nutrition which provides essential elements to gain potential usefulness of food [2]. Proper nutrient intake improves physical performance, on the other hand, nutrient deficiency consequences poor athletic performance $[3,4]$. Healthy dietary pattern and adequate nutrition are necessary for an adolescent's life, especially those who are involved in sports [5]. Previously, only elite athletes were concerned regarding proper nutrition for their performance [6], but now it is understood by all athlete that nutrition is an integral part of their training program $[4,7]$. In adolescent stage, energy requirement increases and varies based on gender and activity [8]; those higher demands are poorly met in adolescent athletes $[5,8,9]$.

In adolescent sports trainee, energy requirements are high for their sport as well as their growing bodies [10]. Therefore, when nutrient intake is inadequate, it leads to poor athletic performance and additional health problems [10]. To avoid illness, injury and to fuel properly in each step (before, during and after) of a sporting event, education on sports nutrition is necessary [8]. Additionally, variation in culture, race, religion, and type of sport leads to variation in nutritional practices [10]. However, several environmental factors (busy schedule, travel and body image) also hinders athletes from getting required dietary practices. Nutrition knowledge also influences attitude and eating behavior [11]. Adolescent sports trainee often relies on their coaches for nutrition guidance despite gaining brief information from their regular textbooks. It creates a potential harm if the coaches are misinformed [12]. This demonstrates the importance of proper nutrition knowledge and healthy dietary practices for adolescent athletes. Therefore, adolescent athletes are the appropriate target group for nutrition education as they have the potential for positive changes [13].

An adolescent from low-income communities lacks educational materials which result in insufficient knowledge of sports nutrition and supplementation to make health conscious decisions. Effect of education on nutrition knowledge and attitude has also not studied well. Though study suggests that short-term education on nutrition can enhance supplementation knowledge [14]. Therefore, the study was designed to find out gaps and areas of knowledge deficits before an intervention to put in place. This study aim to assess nutrition knowledge, attitude and practices of young adolescent athletes and their potential determinants in the context of Bangladeshi.

\section{Methods}

The current study was a cross-sectional study conducted at BKSP (Bangladesh Krira Shikkha Protisthan) among adolescent trainee athletes from the different disciplines of sports. BKSP, known as Bangladesh Institute of Sports, was established in 1976, by the government of Bangladesh. Since then, BKSP is operating as the national sports institute of Bangladesh. It has 17 sports department. The study took place among students of BKSP who train athletes in a different category of sports such as cricket, football, volleyball, and others. The study was conducted from September 2017 to March 2018. The samples were divided into different clusters according to their field of sports. The samples were chosen conveniently from each cluster during the visit after the end of their regular training session. Adolescent trainee athletes participating in different games, aged between 11 to 19 years, were included in this study. Participants had 
more than one year of training in BKSP were also included because newly joined athlete could bias our hypothesis. Participants who were critically ill or diagnosed as malnourished were excluded from this study. Participants who were reluctant to take part were excluded.

\section{Data Collection Technique}

The researchers visited all of the sports departments of BKSP before taking the interview. A short meeting with the corresponding coaches was conducted to discuss the objectives of the study, methodology, and research outcome. After getting permission, a pilot survey was first conducted to revise the designed research tools for the final data collection. A semi-structured questionnaire was used to collect data. Data collection was carried after the daily training session of our target population without hampering their regular activities. The objective of the research was first explained to the athletes verbally, and then the consent to participate in the study was taken.

\section{Questionnaire Design}

A semi-structured interviewer-administered questionnaire was designed for pretesting and necessary modification. The questionnaire consisted of several parts, including demographic information of students, their body composition, knowledge, attitude, and practices towards nutrition. We have designed the first section to get information about the demographic characteristics of students. The second section includes any prior exposure to nutrition training and the sources from where the athletes did get those training and/or information on nutrition. Anthropometric data such as participant's height, weight, BMI, and body fat percentage (\%) was collected. The third section includes the statements to assess the knowledge regarding nutrition, and the fourth section had statements used to evaluate attitude towards nutrition. The questionnaires for the knowledge and attitude section were adapted from a previous study $[15,16]$. The last section includes questions on nutrition practices that were adapted from Folasire et al. [16]. The questionnaires for knowledge, attitude, and practices were then translated into the Bengali language and modified according to the local context. Then proceeded to the pretest and final data collection.

\section{Procedures}

A Stadiometer was used to measure height, maintaining appropriate procedures. Weight was measured using a standard weighing scale. Skinfold caliper was used to measure skinfold thickness of the participants. Participant's BMI, body fat percentage (\%) were calculated using standard formulas. The answer to each question from the questionnaire was taken and recorded.

\section{Knowledge, Attitude, and Practices (KAP) Scoring}

A scoring system was developed to show the results of knowledge, attitude, and practices (KAP) based on the response we have got from the participants. The knowledge section had 21 statements which could be answered as "true" or "false". Each correct response scored as " 1 ," and the incorrect one as " 0 ". In the attitude section, 11 statements were given, which could be answered on a Likert scale ranging from "strongly agree" to "strongly disagree". The scores ranged from " $1-5$ ", where " 5 " was the most positive and 
"1" was the most negative. In the practice section, 15 statements were prepared with their response as "yes" or "no". Each positive response in this section scored as " 1 " and negative responses were scored as" 0 ". After scoring, the score for all of the answered questions for Knowledge, Attitude and Practice was summed up separately. These score means that, the more the score the more the participant have good knowledge score and practice habit and have positive attitude. To decide the individual as having good or bad knowledge score and practice habit and positive or negative attitude towards nutrition, the median score was used as the cutoff point.

\section{Statistical Analysis}

Data were then entered in the Excel sheet and finally analysed using RStudio (R version 3.6.1) and Python Jupyter Notebook. Correlation between Knowledge, Attitude and Practice score were compared using Pearson's correlation coefficient. Means KAP score based on related factors were compared using one-way analysis of variance (ANOVA). Moreover, factors/variables that lead to good or poor KAP score were analyzed using binary logistic regression. Nutrition knowledge, attitude and practices between dichotomous variables, were compared using independent sample t-test. A p-value of $\leq 0.05$ was used as significant for statistical results.

\section{Results}

A total number of 260 adolescent trainee athletes from different sports department of BKSP participated in this research. All the participants were below 19 years of age. The mean \pm SD (Standard Deviation) age of our participant was $15.50 \pm 1.83$ with a range of 12-19 years. Most of the participants were male $(n=224$, $86.2 \%)$, while few were female $(n=36,13.8 \%)$. Moreover, according to the religious category, Muslim $(n=$ $233,89.6 \%)$ were predominant followed by Hindu $(n=21,8.1)$, Christians $(n-5,1.92 \%)$ and Buddhists $(n=1$, $0.38 \%$ ). We did not find any other religions such as tribal group or ethnic people among our participants. Most of the participant were school-going (Secondary school) adolescents $(n=161,61.9 \%)$ followed by the college (Higher Secondary) ( $n=98,37.7 \%)$ and degree or university students $(n=1,0.4 \%)$ (Table 1$)$. Since all students were residents at BKSP, we did not ask them about their house of residence or habitats. 
Table 1

Demographic characteristics of respondents $(n=260)$

\begin{tabular}{|c|c|c|}
\hline Characteristics & Frequency & Percentage \\
\hline Age (15.50 \pm 1.83$)$ years & 131 & 50.4 \\
\hline$\leq 15$ & 129 & 49.6 \\
\hline \multicolumn{3}{|l|}{$>15$} \\
\hline Gender & 233 & 89.62 \\
\hline Male & 27 & 10.38 \\
\hline \multicolumn{3}{|l|}{ Female } \\
\hline Religion & 233 & 89.62 \\
\hline Islam & 21 & 8.08 \\
\hline Hindu & 5 & 1.92 \\
\hline Buddhist & 1 & 0.38 \\
\hline \multicolumn{3}{|l|}{ Christian } \\
\hline BMI Classification (Cut-off) & 53 & 30.4 \\
\hline Underweight $(<18.50)$ & 204 & 78.5 \\
\hline Normal (18.50-24.99) & 3 & 1.2 \\
\hline Overweight $(\geq 25)$ & 0 & 0 \\
\hline \multicolumn{3}{|l|}{ Obese $(\geq 30)$} \\
\hline Educational Level (Current) & 161 & 61.9 \\
\hline High school (Secondary) & 98 & 37.7 \\
\hline College (Higher Secondary) & 1 & 0.4 \\
\hline \multicolumn{3}{|l|}{ Degree/ University (Bachelors) } \\
\hline \multicolumn{3}{|l|}{ Department of Training } \\
\hline Cricket & 42 & 16.2 \\
\hline Football & 36 & 13.8 \\
\hline Athletics & 37 & 14.2 \\
\hline Basketball & 25 & 9.6 \\
\hline Volleyball & 20 & 7.7 \\
\hline Archery & 27 & 10.4 \\
\hline
\end{tabular}




\begin{tabular}{|lll|}
\hline Characteristics & Frequency & Percentage \\
\hline Table Tennis & 21 & 8.1 \\
\hline Karate & 10 & 3.8 \\
Wushu & 14 & 5.4 \\
\hline Taekwondo & 8 & 3.1 \\
\hline Hockey & 20 & 7.7 \\
\hline
\end{tabular}

BKSP has 17 distinct departments where athletes were being trained according to their capacity and guidance from the coach. In this research, we have collected information from 11 departments following the inclusion criteria using a convenient sampling technique. Most of our participants were from the department of Cricket $(n=42,16.2 \%)$ followed by Athletic $(n=37,14.2 \%)$, Football $(n=36,13.8 \%)$, Archery $(n=27,10.4 \%)$ and so forth. Due to the limitation of time, we could not reach all the departments. The number of participants, according to the departments of sports, are shown in Table 1.

Participants who had their training period in BKSP of 1 or more than 1 year were only included in the study. The mean \pm SD (Standard Deviation) of their training was $3.67 \pm 2.29$ years ranging from 1 to 13 years. Most of our participants were young trainee athletes (Table 2). Majority of our participants had their training period in their corresponding sports department equal to 5 years or less $(n=206,79.2 \%)$. Nearly 21 per cent of respondents received training for more than five years. 
Table 2

Nutrition education among survey respondents $(n=260)$

\begin{tabular}{|lll|}
\hline & Frequency & Percentage \\
\hline Number of nutrition training & 15 & 5.8 \\
Once & 6 & 2.3 \\
Twice & 6 & 2.3 \\
Thrice & 0 & 0 \\
$\geq 4$ th time & 233 & 89.6 \\
Never & & \\
\hline Duration of Training (Years) & 206 & 79.2 \\
$\leq 5$ & 54 & 20.8 \\
$>5$ & & \\
\hline Time since last training was received $(n=27)$ & 3 & 1.2 \\
1 month ago & 10 & 3.8 \\
3 months ago & 8 & 3.1 \\
6 months ago & 6 & 2.3 \\
1 year ago & & \\
\hline
\end{tabular}




\begin{tabular}{|lll|}
\hline & Frequency & Percentage \\
\hline Source of Information & 97 & 37.3 \\
Book & 5 & 1.9 \\
Magazine & 9 & 3.5 \\
Newspaper & 27 & 10.4 \\
Teachers & 78 & 30.0 \\
Coach & 3 & 1.2 \\
Trainer & 7 & 2.7 \\
Doctor & 13 & 5.0 \\
Nutritionist & 10 & 3.8 \\
Parents & 4 & 1.5 \\
Teammates & 6 & 2.3 \\
Internet & 1 & 0.4 \\
Others & 0 & 0 \\
No source & & \\
\hline
\end{tabular}

\section{Anthropometric Details}

The mean \pm SD weight of our respondents was $57.31 \pm 9.41$ kilograms ranging from 25 to 83 kilograms. The mean \pm SD (Standard Deviation) height of the participants was $5.47 \pm 0.38$ feet ranging from 4.10 to 6.50 feet. In this study, the mean \pm SD (Standard Deviation) BMI was $20.07 \pm 2.05$ ranging from 13.80 to 25.70. Most of our participants belong to the healthy category of BMI $(n=204,78.5 \%)$. Around one-third of the participant were underweight $(n=53,30.4 \%)$, however only $3(1.2 \%)$ were overweight and no obese individuals (Table 1). The mean \pm SD (Standard Deviation) body fat percentage (\%) of all respondents was $9.06 \pm 4.59$ ranging from 4.10 to 25 .

\section{Nutrition Education}

Participants were asked if they had any previous training or attended in seminar/workshop on nutrition. Round 9 out of 10 participants replied that they never had any nutritional education or training during their sports training period $(n=233,89.6 \%)$. Only 10 percent replied of positive of having nutritional training before. Participants, who had prior nutrition training, were further asked details about training like them how long ago they had their last training or education in nutrition. Only 27 participants $(10.4 \%)$ had 
previous exposure to nutrition training; among them, only ten participants had their training three months ago. Six of them have had their previous nutrition training one year before (Table 2).

\section{Source Of Information On Nutrition}

Participants were asked about their most common source of information regarding nutritional knowledge and dietary practices. Nearly forty per cent of respondents replied that they got information about nutrition from the textbooks ( $n=97,37.3 \%$ ). Thirty per cent of them replied that coaches were the primary source of information ( $n=78)$, followed by teachers $(n=27,10.4 \%)$. Very few of them have mentioned about other reliable sources such as doctors, nutritionists, trainers, health magazines. Details distribution hare shown in Table 2.

\section{Nutrition Knowledge}

The mean \pm Standard Deviation (SD) nutrition knowledge score for the entire sample was $12.77 \pm 2.17$ (Fig. 1). The highest score was 18, while the lowest score was 5 . The knowledge scores were divided into two categories as good and poor based on the median score of the participants. More than half of the participants had good nutrition knowledge $(n=149,57.3 \%)$ whereas nearly forty-three percent of respondents had reduced knowledge level on nutrition ( $n=111,42.7 \%)$.

Age had a significant relationship with nutrition knowledge score (Table 3). Participants, more than 15 years of age, had better level of knowledge than their juniors $(p=0.007)$. Their mean \pm Standard Deviation (SD) knowledge score was $13.13 \pm 2.13$, which was higher than the other age group $(<15$ years) (Table 3). Boys had significantly better nutrition knowledge than girls $(p=0.004$ with an average knowledge score of $12.92 \pm 2.12$ (Table 3 ). The educational level of the participants did not show any significant influence on their nutritional knowledge. Participants who were studying in schools has a similar level of mean knowledge score with those who are studying in college $(p=0.619)$. There was also a significant relationship between knowledge score, and the department of training $(p=0.003)$. Cricket, Football, and Wushu trainee adolescents had a relatively higher level of knowledge on nutrition than the other group of participants. 
Table 3

Bivariate Analysis of Nutrition Knowledge, Attitude and Practices $(n=260)$

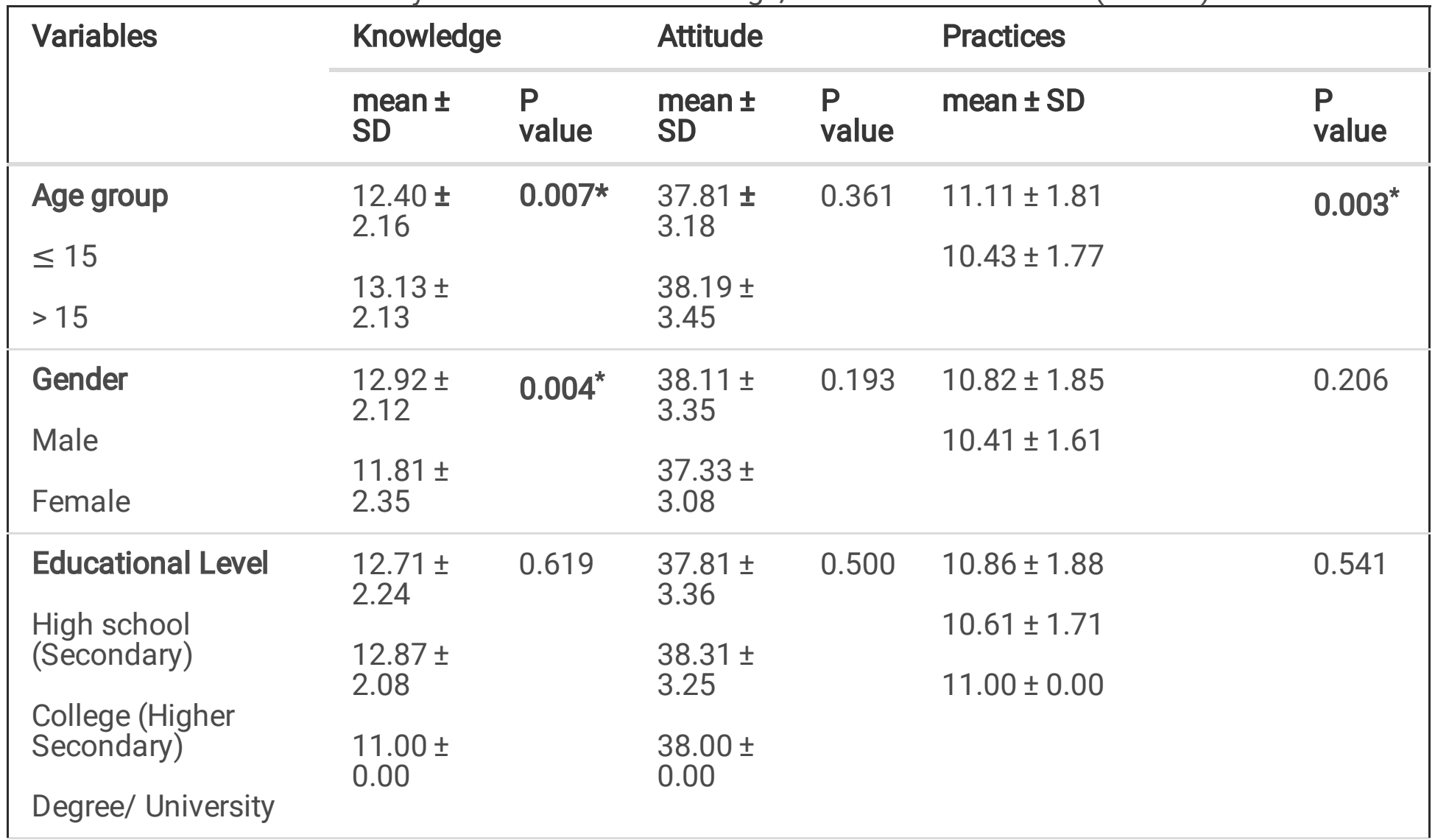




\begin{tabular}{|c|c|c|c|c|c|c|}
\hline \multirow[t]{2}{*}{ Variables } & \multicolumn{2}{|c|}{ Knowledge } & \multicolumn{2}{|l|}{ Attitude } & \multicolumn{2}{|l|}{ Practices } \\
\hline & $\begin{array}{l}\operatorname{mean} \pm \\
\mathrm{SD}\end{array}$ & $\begin{array}{l}P \\
\text { value }\end{array}$ & $\begin{array}{l}\operatorname{mean} \pm \\
\mathrm{SD}\end{array}$ & $\begin{array}{l}P \\
\text { value }\end{array}$ & mean $\pm S D$ & $\begin{array}{l}P \\
\text { value }\end{array}$ \\
\hline $\begin{array}{l}\text { Department of } \\
\text { Training }\end{array}$ & $\begin{array}{l}11.9 \pm \\
1.80\end{array}$ & \multirow[t]{11}{*}{0.0034} & $\begin{array}{l}38.2 \pm \\
3.47\end{array}$ & \multirow[t]{11}{*}{0.929} & $10.7 \pm 1.54$ & \multirow[t]{11}{*}{0.256} \\
\hline Archery & $\begin{array}{l}12.4 \pm \\
2.67\end{array}$ & & $\begin{array}{l}38.5 \pm \\
3.54\end{array}$ & & $10.7 \pm 1.81$ & \\
\hline Athletics & $\begin{array}{l}12.0 \pm \\
1.86\end{array}$ & & $\begin{array}{l}37.8 \pm \\
2.68\end{array}$ & & $\begin{array}{l}10.6 \pm 1.87 \\
11.1 \pm 1.81\end{array}$ & \\
\hline $\begin{array}{l}\text { Cricket } \\
\text { Football }\end{array}$ & $\begin{array}{l}13.5 \pm \\
1.98\end{array}$ & & $\begin{array}{l}37.5 \pm \\
3.07\end{array}$ & & $11.0 \pm 1.17$ & \\
\hline $\begin{array}{l}\text { Football } \\
\text { Hockey }\end{array}$ & $\begin{array}{l}13.0 \pm \\
1.80\end{array}$ & & $\begin{array}{l}37.8 \pm \\
3.62\end{array}$ & & $\begin{array}{l}9.6 \pm 1.35 \\
11.2 \pm 1.81\end{array}$ & \\
\hline $\begin{array}{l}\text { Karate } \\
\text { Table Tennis }\end{array}$ & $\begin{array}{l}12.9 \pm \\
1.83\end{array}$ & & $\begin{array}{l}38.1 \pm \\
4.46\end{array}$ & & \multirow{2}{*}{$\begin{array}{l}10.8 \pm 1.9111 .0 \pm 2.00 \\
9.79 \pm 2.04\end{array}$} & \\
\hline Taekwondo & $\begin{array}{l}12.6 \pm \\
2.32\end{array}$ & & $\begin{array}{l}37.3 \pm \\
3.27\end{array}$ & & & \\
\hline \multirow{4}{*}{$\begin{array}{l}\text { Volleyball } \\
\text { Wushu }\end{array}$} & $\begin{array}{l}12.8 \pm \\
2.14\end{array}$ & & $\begin{array}{l}38.0 \pm \\
3.02\end{array}$ & & & \\
\hline & $\begin{array}{l}12.9 \pm \\
1.46\end{array}$ & & $\begin{array}{l}37.4 \pm \\
2.00\end{array}$ & & & \\
\hline & $\begin{array}{l}12.2 \pm \\
2.80\end{array}$ & & $\begin{array}{l}38.9 \pm \\
1.86\end{array}$ & & & \\
\hline & $\begin{array}{l}14.6 \pm \\
1.70\end{array}$ & & $\begin{array}{l}38.3 \pm \\
4.61\end{array}$ & & & \\
\hline
\end{tabular}




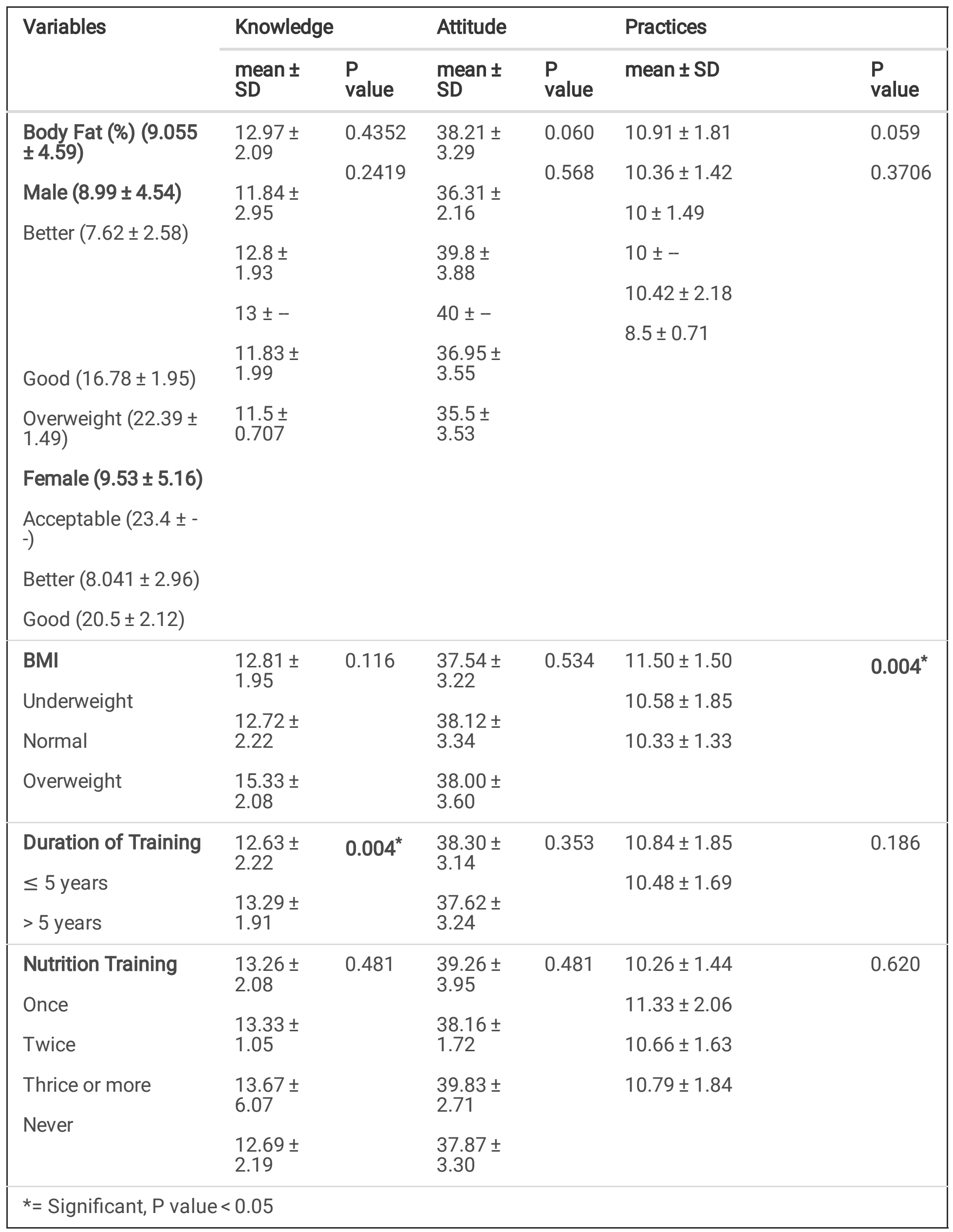


BMI of our participant was not significantly corelated with their nutrition knowledge $(p=0.116)$ (Table 3$)$. Nutrition knowledge score was not significantly different among participants with different level of different body fat percentage and this level of insignificant was also true for both male and female athletes as well (Table 3$)(0.4352$ and $p=0.2419)$. Duration of training received by participant had a significant relationship with the nutrition knowledge score $(p=0.044)$. Participants with more than five years of training had a higher level of knowledge in nutrition than the participant who had less than or equal to 5 years of training. Whereas, previous training on nutrition did not have any significant influence on the nutrition knowledge of our respondents $(p=0.481)$ (Table 3$)$.

\section{Attitude Towards Nutrition}

The questionnaire consisted of 11 statements concerning attitudes toward nutrition; the highest possible score was 55 while the lowest possible score was 11 . The mean \pm Standard Deviation (SD) score for attitude towards nutrition for the entire sample was $38.00 \pm 3.32$. The highest score was 46 , whereas the lowest score was 28 . The attitude score was also divided into two categories, such as positive and negative, based on the median value (Fig. 1). Less than half of our participants had a positive attitude towards nutrition ( $n=114,43.8 \%$ ), while fifty-six per cent of participants had a negative attitude towards nutrition ( $n=146)$ (Fig. 1).

Unlike knowledge score, attitude score was not significantly different among participants of different age group $(p=0.361$, Table 3$)$. Though participants more than 15 years of age had a better level of attitude score than their juniors. Moreover, boys had a better attitude towards nutrition than girls, but the finding was not statistically significant $(p=0.193)$. The educational level of the participants did not have any influence on their attitude towards nutrition. A participant who is studying in college had a higher level of mean attitude score than those who are studying in schools, but the relationship was not statistically significant $(p=0.500)$. There was no significant relationship between attitude score and department of training $(p=0.929)$. BMI of our participant also did not show any significant relationship with their attitude towards nutrition $(p=0.534)$. Body fat percentage (BF \%) and attitude score also follow the similar insignificant relationship $(p=0.902)$. Moreover, the duration of training received by participants and attitude scores shown to have insignificant relation $(p=0.353)$. Previous training on nutrition did not have any significant influence on attitude towards nutrition of our respondents $(p=0.225)$ (Table 3$)$.

\section{Nutrition Practices}

The maximum practice score was 15 , while the minimum was 6 , and the practice score was calculated based on 15 statements on nutrition practice. The mean \pm Standard Deviation (SD) of practice score was $10.77 \pm 1.82$. Practice score was also divided into two categories, such as good or poor and this categorization was also done based on the median score. More than half of the athletes had good level of practices $(n=150,57.7 \%)$ while $42.3 \%(n=110)$ had the practice score level poor (Fig. 1). 
Age had a significant relationship with nutrition practice score of our participants. Participants, less than 15 years of age, had a better level of practices than their seniors which was significant statistically $(p=$ $0.003)$. In this research, we did not find any significant difference in nutrition practices between male and female participants $(p=0.206)$. The educational level of the participants did not have any influence on their practices of nutrition $(p=0.541)$. Moreover, there was no significant relationship between practice score and department of training $(p=0.256)$. BMI of our participant had a significant relationship with their practices of nutrition $(p=0.004)$. We didn't find any significant correlation between body fat percentage (BF $\%)$ and practice score of the participants ( $(p=0.059$ for male and $p=0.3706$ for female). We did not find any significant relationship between duration of training received by participants with the nutrition practice score $(p=0.186)$. Previous training on nutrition also did not have any significant influence on practice towards nutrition among our respondents $(p=0.620)$. Table 4 summarizes the detail.

Table 4: Unadjusted and Adjusted Odd Ratio of the association of different variables with knowledge, attitude and practice score. 
Knowledge Score

(Good vs Poor)
Attitude Score

(Positive vs Negative)
Practice Score

(Good vs Poor)

\begin{tabular}{|c|c|c|c|c|c|c|}
\hline Factors & $\begin{array}{l}\text { Unadjusted } \\
\text { OR }(95 \% \mathrm{Cl})\end{array}$ & $\begin{array}{l}\text { Adjusted } \\
\text { OR }(95 \% \mathrm{Cl})\end{array}$ & $\begin{array}{l}\text { Unadjusted } \\
\text { OR (95\%Cl) }\end{array}$ & $\begin{array}{l}\text { Adjusted } \\
\text { OR } \\
(95 \% \mathrm{Cl})\end{array}$ & $\begin{array}{l}\text { Unadjusted } \\
\text { OR }(95 \% \mathrm{Cl})\end{array}$ & $\begin{array}{l}\text { Adjusted } \\
\text { OR }(95 \% \mathrm{Cl})\end{array}$ \\
\hline $\begin{array}{l}\text { Gender } \\
\text { Female } \\
\text { (Ref) } \\
\text { Male }\end{array}$ & $\begin{array}{l}1 \\
2.11(0.94- \\
4.75)\end{array}$ & $\begin{array}{l}1 \\
2.037 \\
(0.847- \\
4.901)\end{array}$ & $\begin{array}{l}1 \\
1.51(0.68- \\
3.36)\end{array}$ & $\begin{array}{l}1 \\
1.676 \\
(0.715- \\
3.929)\end{array}$ & $\begin{array}{l}1 \\
2.56 \\
(1.12- \\
5.83)^{\star}\end{array}$ & $\begin{array}{l}1 \\
2.91(1.149- \\
7.373) *\end{array}$ \\
\hline $\begin{array}{l}\text { Age } \\
\geq 15 \text { vs }< \\
15\end{array}$ & $\begin{array}{l}1.78(1.08- \\
2.92)^{\star}\end{array}$ & $\begin{array}{l}1.119 \\
(0.594- \\
2.106)\end{array}$ & $\begin{array}{l}1.12(0.69- \\
1.86)\end{array}$ & $\begin{array}{l}0.996 \\
(0.529- \\
1.875)\end{array}$ & $\begin{array}{l}0.59 \\
(0.36- \\
0.96)\end{array}$ & $\begin{array}{l}0.609 \\
(0.316- \\
1.175)\end{array}$ \\
\hline Weight (kg) & $\begin{array}{l}1.02(0.99- \\
1.05)\end{array}$ & $\begin{array}{l}1.59(1.14- \\
2.207)^{\star}\end{array}$ & $\begin{array}{l}1.02 \\
(0.99- \\
1.05)\end{array}$ & $\begin{array}{l}0.962 \\
(0.733- \\
1.262)\end{array}$ & $\begin{array}{l}0.98(0.95- \\
1.0)\end{array}$ & $\begin{array}{l}1.021(0.75- \\
1.391)\end{array}$ \\
\hline $\begin{array}{l}\text { Height } \\
\text { (Inch) }\end{array}$ & $\begin{array}{l}0.99(0.93- \\
1.05)\end{array}$ & $\begin{array}{l}0.489(0.291- \\
0.822)^{\star}\end{array}$ & $\begin{array}{l}1.05 \\
(0.99- \\
1.12)\end{array}$ & $\begin{array}{l}1.131 \\
(0.735- \\
1.742)\end{array}$ & $\begin{array}{l}0.97 \\
(0.92- \\
1.03)\end{array}$ & $\begin{array}{l}0.887 \\
(0.536- \\
1.466)\end{array}$ \\
\hline BMI & $\begin{array}{l}1.12(1.0- \\
1.24)^{\star}\end{array}$ & $\begin{array}{l}0.31(0.126- \\
0.766)^{\star}\end{array}$ & $\begin{array}{l}1.02 \\
(0.92- \\
1.14)\end{array}$ & $\begin{array}{l}1.129 \\
(0.529- \\
2.411)\end{array}$ & $\begin{array}{l}0.92 \\
(0.82- \\
1.02)\end{array}$ & $\begin{array}{l}0.926 \\
(0.386- \\
2.225)\end{array}$ \\
\hline $\begin{array}{l}\text { Body Fat } \\
(\%)\end{array}$ & $\begin{array}{l}0.97(0.92- \\
1.03)\end{array}$ & $\begin{array}{l}0.967 \\
(0.904- \\
1.035)\end{array}$ & $\begin{array}{l}1.01 \\
(0.95- \\
1.06)\end{array}$ & $\begin{array}{l}1.019 \\
(0.953- \\
1.089)\end{array}$ & $\begin{array}{l}0.9(0.85- \\
0.95)^{\star}\end{array}$ & $\begin{array}{l}0.895(0.83- \\
0.96) *\end{array}$ \\
\hline $\begin{array}{l}\text { Dept. of } \\
\text { Training } \\
\text { Cricket } \\
\text { (Ref) }\end{array}$ & 1 & 1 & 1 & 1 & 1 & 1 \\
\hline Football & $\begin{array}{l}0.79 \\
(0.31,1.99)\end{array}$ & $\begin{array}{l}0.63(0.23- \\
1.727)\end{array}$ & $\begin{array}{l}0.76 \\
(0.31- \\
1.87)\end{array}$ & $\begin{array}{l}0.778 \\
(0.302- \\
2.006)\end{array}$ & $\begin{array}{l}2.36 \\
(0.92-6.1)\end{array}$ & $\begin{array}{l}1.694(0.602- \\
4.7679)\end{array}$ \\
\hline Wushu & $\begin{array}{l}3.0 \\
(0.59,15.29)\end{array}$ & $\begin{array}{l}2.21(0.405- \\
12.058)\end{array}$ & $\begin{array}{l}1.22 \\
(0.35- \\
4.29)\end{array}$ & $\begin{array}{l}1.12 \\
(0.304- \\
4.117)\end{array}$ & $\begin{array}{l}0.91 \\
(0.27- \\
3.05)\end{array}$ & $\begin{array}{l}1.051 \\
(0.291- \\
3.796)\end{array}$ \\
\hline Hockey & $\begin{array}{l}0.75 \\
(0.25,2.26)\end{array}$ & $\begin{array}{l}0.508 \\
(0.154- \\
1.674)\end{array}$ & $\begin{array}{l}1.02 \\
(0.34- \\
3.02)\end{array}$ & $\begin{array}{l}0.939 \\
(0.302- \\
2.921)\end{array}$ & $\begin{array}{l}2.12 \\
(0.68- \\
6.58)\end{array}$ & $\begin{array}{l}2.045 \\
(0.601- \\
6.961)\end{array}$ \\
\hline Archery & $\begin{array}{l}0.46 \\
(0.17,1.25)\end{array}$ & $\begin{array}{l}0.571(0.2 \\
-1.634)\end{array}$ & $\begin{array}{l}0.99 \\
(0.37- \\
2.65)\end{array}$ & $\begin{array}{l}1.058 \\
(0.382- \\
2.929)\end{array}$ & $\begin{array}{l}0.979 \\
(0.372- \\
2.5768)\end{array}$ & $\begin{array}{l}1.003 \\
(0.349- \\
2.883)\end{array}$ \\
\hline Athletics & $\begin{array}{l}0.59 \\
(0.24,1.46)\end{array}$ & $\begin{array}{l}0.61(0.231- \\
1.611)\end{array}$ & $\begin{array}{l}0.89 \\
(0.36- \\
2.19)\end{array}$ & $\begin{array}{l}0.843 \\
(0.332- \\
2.14)\end{array}$ & $\begin{array}{l}1.07 \\
(0.44- \\
2.59)\end{array}$ & $\begin{array}{l}1.17(0.445- \\
3.075)\end{array}$ \\
\hline
\end{tabular}




\begin{tabular}{|c|c|c|c|c|c|c|}
\hline \multirow[b]{2}{*}{ Basketball } & \multicolumn{2}{|c|}{$\begin{array}{l}\text { Knowledge Score } \\
\text { (Good vs Poor) }\end{array}$} & \multicolumn{2}{|c|}{$\begin{array}{l}\text { Attitude Score } \\
\text { (Positive vs Negative) }\end{array}$} & \multicolumn{2}{|c|}{$\begin{array}{l}\text { Practice Score } \\
\text { (Good vs Poor) }\end{array}$} \\
\hline & $\begin{array}{l}0.39 \\
(0.14,1.09)\end{array}$ & $\begin{array}{l}0.18(0.045- \\
0.71)^{\star}\end{array}$ & $\begin{array}{l}0.87 \\
(0.32- \\
2.36)\end{array}$ & $\begin{array}{l}0.617 \\
(0.176- \\
2.1681)\end{array}$ & $\begin{array}{l}1.16 \\
(0.43- \\
3.13)\end{array}$ & $\begin{array}{l}1.171 \\
(0.324- \\
4.238)\end{array}$ \\
\hline Karate & $\begin{array}{l}0.5 \\
(0.12,2.02)\end{array}$ & $\begin{array}{l}0.471(0.11- \\
2.016)\end{array}$ & $\begin{array}{l}0.45 \\
(0.11- \\
1.85)\end{array}$ & $\begin{array}{l}0.457 \\
(0.109- \\
1.92)\end{array}$ & $\begin{array}{l}0.23 \\
(0.04-1.2)\end{array}$ & $\begin{array}{l}0.278 \\
(0.049- \\
1.588)\end{array}$ \\
\hline $\begin{array}{l}\text { Table } \\
\text { Tennis }\end{array}$ & $\begin{array}{l}0.67 \\
(0.23,1.96)\end{array}$ & $\begin{array}{l}0.503 \\
(0.154- \\
1.648)\end{array}$ & $\begin{array}{l}1.1(0.38- \\
3.24)\end{array}$ & $\begin{array}{l}1.55 \\
(0.475- \\
5.055)\end{array}$ & $\begin{array}{l}1.48 \\
(0.51-4.3)\end{array}$ & $\begin{array}{l}1.498 \\
(0.448-5.01)\end{array}$ \\
\hline Taekwondo & $\begin{array}{l}0.5 \\
(0.11,2.3)\end{array}$ & $\begin{array}{l}0.501 \\
(0.105- \\
2.393)\end{array}$ & $\begin{array}{l}0.1(0.01- \\
0.86)^{\star}\end{array}$ & $\begin{array}{l}0.095 \\
(0.011- \\
0.856)^{\star}\end{array}$ & $\begin{array}{l}1.52 \\
(0.32- \\
7.17)\end{array}$ & $\begin{array}{l}1.057 \\
(0.213- \\
5.241)\end{array}$ \\
\hline Volleyball & $\begin{array}{l}0.5 \\
(0.17,1.48)\end{array}$ & $\begin{array}{l}0.311 \\
(0.085- \\
1.142)\end{array}$ & $\begin{array}{l}2.04 \\
(0.62- \\
6.67)\end{array}$ & $\begin{array}{l}1.563 \\
(0.419- \\
5.83)\end{array}$ & $\begin{array}{l}1.69 \\
(0.56- \\
5.07)\end{array}$ & $\begin{array}{l}1.88(0.52- \\
6.7)\end{array}$ \\
\hline $\begin{array}{l}*=\text { Significa } \\
0.05\end{array}$ & alue < & & & & & \\
\hline
\end{tabular}

\section{Determinants Of Nutrition Knowledge, Attitude And Practice}

The determinants of nutrition knowledge, attitude and practice score of athletes are shown in Table 3 and Table 4. Bivariate analysis shows that knowledge score was significantly different between athletes categorized according to age, gender and duration of training (Table 3). Whereas, none of the category of athletes show significant difference of attitude score and practice score was only shown to have significant difference between/among athletes categorize according to age and BMI.

Multivariate logistic regression shows significant effect of age, weight, height, BMI and dept. of training on knowledge score. Athletes $\geq 15$ years of age had greater chances of having good nutrition knowledge score (UAOR 1.119; $95 \% \mathrm{Cl} 1.08,2.92 ; \mathrm{p}<0.05)$ compared to athletes $<15$ years of age. However, after adjusting other variables, effect of age category became insignificant $(p>0.05)$. One $\mathrm{kg}$ increase in weight leads to 1.59-fold increase of odd of having good nutrition knowledge score (AOR 1.59; 95\% Cl 1.14, 2.207; $\mathrm{p}<0.05)$. Unlike weight, every inch increase in height decreases the chance of having good knowledge score (AOR 0.489; 95\% Cl 0.291, 0.822; $\mathrm{p}<0.05$ ). Increase in BMI also follow the same decreasing chance

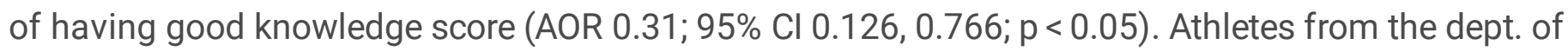
Karate shown to have around half of the odd of having good knowledge score (AOR $0.18 ; 95 \% \mathrm{Cl} 0.045$, $0.071 ; p<0.05)$ compared to athletes from the dept. of cricket. 
None of the variables shown to have significant impact on the attitude score. Whereas, when practicing good nutrition habit, male was shown have around 3 times more odd of good practice score compared to the female counterpart (AOR $2.91 ; 95 \% \mathrm{Cl} 1.149,7.373 ; \mathrm{p}<0.05$ ). Athletes with more body fat was more likely to have less practice score (AOR $0.895 ; 95 \% \mathrm{Cl} 0.83,0.96 ; p<0.05$ ). As the body fat percentages of athletes decreases the predicted probability of having good practice score also increases and both male and female athletes follow this trend,

\section{The relationship between Knowledge and Attitude Score with Practices Score}

The logistic regression model was developed to predict, how knowledge and attitude score increase or decrease the chance of having good practice score and shown in the Fig. 4. Athletes, those having good knowledge score were more than 2 times likely to have good practice score (AOR 2.335; 95\% Cl 1.405, 3.88; $p=0.001)$. Predicted probability of good practice score of athletes also increases as the knowledge score increases and this trend was true for both male and female athletes (Fig. 3). Attitude score didn't show any positive of negative impact on practice score.

\section{Discussion}

Intake of adequate nutrition enhances athletic performance. The extent of exercise and intensity of training of an athlete also determines his/her nutritional requirements. Nutrition is an essential and integral part of a training program for proper fueling through optimal nutrition; most of the athletes nowadays understand this fact [17]. However, most of them also lack proper knowledge and healthy dietary practices [6]. Therefore, this study investigated the current knowledge, attitude, and practices towards nutrition, the relationship between each other among adolescent athletes of BKSP, Bangladesh.

From the result, we have found that the nutrition knowledge of our respondents was insufficient. The mean knowledge score was similar to another study which had the same knowledge statements [15]. Inadequate knowledge was also observed in some other related studies [10]. Male participants had a higher level of nutrition knowledge score than females in a study [18]. The comparison was not statistically significant reported by another study [19]. Nevertheless, in our study, we have found a significant difference between genders in nutrition knowledge score. Males had better knowledge score (Table 3) and more likely to have good knowledge score (Table 4) than females, however that was not statistically significant. This insignificant difference of knowledge score between male and female athletes also reported by few other studies $[20,21]$. Athletes more than 15 years of age had better knowledge score compared to athletes less than 15 years of age (Table 3). However, the odd of having good nutrition knowledge was not significant among the older athletes than the younger (Table 4). Nevertheless, the crude odd ratio show, older athletes are more likely to have good nutrition knowledge. This adjustment effect could be explained as the effect of other variables included in the regression model. Rikrak Ch Marak et al. (2018) reported no differences of nutrition knowledge between older and younger athletes [20]. However, older athletes are more likely to have extra nutrition training or education compared to the younger. Heikkila, Maria, et al. (2018) also reported the similar effect of age on nutrition knowledge [22]. Athletes were more likely of having good nutrition knowledge with increasing their BMI (Table 4 and Fig. 2). Nonetheless, when adjusting other 
variables, the relation reversed and athletes with lower BMI were more likely of having good nutrition knowledge. This may be due the effect of other variables. Nutrition training had a significant association with participant's knowledge in some studies $[16,19]$. In this study, the mean knowledge score was higher among those participants who had previous exposure to nutrition training, but the difference was not significantly associated. $(p>0.05)$. The reason could be a vast number of participants in this study did not have any formal nutrition education before.

In this study, the primary source of information for nutrition were books, teachers, and coaches (Table 2). Very few of them sought nutrition advice from nutritionist/ dietician. The result was inconsistent with other studies, where most athletes preferred athletic trainers than dieticians as to access nutrition-related information [23, 24]. Magazines, parents, teachers, and teammates were the commonly cited sources for nutrition revealed by another study [16] which is also similar to our results. However, such sources may not always be reliable. The quality of nutrition is vital than the number of available sources. This may also have been responsible for poor nutritional knowledge and routine dietary practices of athletes. To guide athletes better, the importance of coaches and trainers having appropriate nutrition information was emphasized [25]. Duration of the training period had a significant association with nutrition knowledge. Participants with long duration of training scored better than their comparison group.

In the attitude section, the majority of them had a positive attitude towards nutrition, though the percentage is not that high. The mean score was relatively lower than a similar study having similar attitude statements [19]. We did not find any significant differences between age group, gender, educational level, BMI categories, and duration of the training and previous exposure to nutrition training. This finding was following some other related study [19]. Though another study had reported that females had higher attitude score than their counterpart [26]. Nutrition knowledge had a significantly positive correlation with nutrition attitude reported by many studies $[6,16,19,26-28]$. The finding was following our finding.

Reduced level of practices was also observed in most of our respondents, which were in accordance with another study having the same practice statements [10]. Nutrition knowledge was positively and significantly correlated with nutrition practices in this study. The result was in agreement with other previous studies [6, 10], but in contrast with another study [29]. Therefore, it is evident that proper education on nutrition not only increases knowledge but also have positive impacts on practices. Age had a significant relationship with nutrition knowledge and practices. Participants with higher age group had a better level of knowledge, but participants with lower age group in this study had a good level of practices. The reason could be lower age group participants tend to follow the diet schedule maintained at their residence. Nutrition knowledge, attitude, and practices improve with age except for the elderly observed by many other studies [30,31]. This may be due to their education, extended presence in their respective training department and life-related experiences. We did not observe any gender-based differences in nutrition practices. Our finding was also supported by other similar studies where there was no significant difference in nutrition-related behaviors [11,32]. It is often thought that females tend to be more conscious 
and knowledgeable regarding diet/ nutrition-related issues [30], but this is may not be the case in this study as our target population was adolescent athletes.

In the current study, athletes higher body fat tends to practice nutrition guidelines less compared to those with lower body fat percentages. This is a clear indication that athletes with lower body fat tend to practice more or otherwise athletes those who practice more were able to keep their body fat minimum. However, nutrition knowledge and attitude were not related significantly with body fat percentages. This phenomenon could be explained that though athletes belongs limited nutrition knowledge and negative attitude, practice more to keep fit for better performance. Moreover, the predicted probability of practicing more was higher among athletes with lower body fat percentages. In addition, when shedding light on relation between knowledge score and practice score, the odd and predicted probabilities of higher practice score was higher among athletes with higher knowledge score. This finding is in concur with some previous studies; however, the correlation was not strong [33-36]. Thus, the current findings indicate that's nutrition knowledge is positively associated with nutrition practice and modulating factors like duration of training could be a focal point of enchaining nutrition practice behavior. The other factors like age, gendera and height could have little opportunity to change. Moreover, few of these factors, like weight, height and $\mathrm{BMI}$, are inversely and ultimately dependent on good nutrition knowledge and practice behavior of athletes,

The main limitation of this study was the small sample size and a particular age group. Moreover, dipropionate number of athletes from different category of variables, like gender, religious group, BMI category, education level category and so forth, pose potential problems in statistical validity. Another limitation was the use of the questionnaire; social desirability may diminish the validity of the responses. The main strength was the samples included from a variety of sports department from BKSP. As our target populations are sports trainee, most of the participants had a healthy level of BMI. If a large number of adolescents from other schools who are underweight/ overweight (not athletes) could be included, the result could have differed. Moreover, the dipropionate number of athletes from each of the strata, like sports department, receiving training session, pose problem in statistical analysis. Further, research, including analysis of specific food habits and the relationship between diet and diseases can help to determine appropriate nutritional education intervention in the long-term effect on health and athletic performance. Additionally, owing to a limited number of nutrition-related studies in Bangladesh, we suggest conducting more interventional studies to contribute to the promotion of nutrition education, aiming at improving athlete's performance. Moreover, the findings of this study could be useful to generalize for athletes from different area of Bangladesh, if further research was conducted ensuring large sample consisting proportionate number of athletes from different sports department and ensuring inclusion of assessing nutrition knowledge of professional sportsperson or coaches.

\section{Conclusion}

Both coaches and athletes lack sufficient knowledge of nutrition that can provide them such environment resulting in enhanced their performance and ideal health [11]. In this research, the poor level of knowledge, negative attitude, and poor practices were observed in most of the respondents. Age, gender and duration 
of sports training received were the significantly associated variable for nutrition knowledge, while only age and BMI were [37] significantly associated variable for nutrition practices. For attitude towards nutrition, there were no such variables that had a significant association. Nutrition knowledge is significantly correlated with attitude and practices. We can conclude that promotion of nutrition knowledge subsequently improves the attitude and practices. An appropriate diet has a considerable effect on athletic performance. Therefore, the authority should pay attention to the nutrition issues revealed in this study. Regular education session (workshops, courses, seminars) for students by experts, including nutrition courses in the curriculum and coaches' education on nutrition, are some of the key recommendations based on the results in this study. This emphasizes the need for the development and collaboration of sports nutritionist/ dietician in each sports department to bring positive changes in this area.

\section{Abbreviations}

BKSP: Bangladesh Krira Shikkha Protishtan; KAP: Knowledge, Attitude, and Practices; BMI: Body Mass Index; BF: Body Fat; ANOVA: Analysis of Variance; SD: Standard Devia-tion; UAOR: Un-Adjusted Odd Ratio; AOR: Adjusted Odd Ratio; Cl: Confidence Interval.

\section{Declarations}

\section{Ethics approval and consent to participate}

This study was approved by the ethical review board of the faculty of Biological Science of Islamic University, Kushtia, Bangladesh. All participants provided written informed consent prior to their participation in the study. Written informed consent for participants under 16 years old were taken from the respected coaches of each sport department.

\section{Consent for publication}

All authors provide consent for publication of this paper.

\section{Availability of data and material}

The datasets used and/or analysed during the current study are available from the corresponding author on reasonable request.

\section{Competing interests}

The authors declare that they have no competing interests.

\section{Funding}

Not applicable.

\section{Authors' contributions}


The study was designed by MB, MMUR, MK and SSR. Data was collected by MB, MMUR, NS and KSRahaman. Data analysis, interpretation and manuscript preparation was undertaken by MK. All authors read and approved the final manuscript.

\section{Acknowledgments}

The authors would like to thank the BKSP authority and coaching staff of all of the training department for their cooperation.

\section{Author details}

${ }^{1}$ Senior Research Officer, Department of Exercise Physiology, BKSP, Zirani, Savar, Dhaka. ${ }^{2}$ Clinical Physiotherapist, Protibondhi Sheba o Sahahajjo Kendro Magura, Ministry of Social Welfare, Bangladesh. ${ }^{3}$ Medical Officer (Homeopathy), 250 Bedded General Hospital, Meherpur, Bangladesh. ${ }^{4}$ Public Health Specialist, Good Neighbors Bangladesh, Dhaka, Bangladesh. ${ }^{5}$ Department of Applied Nutrition and Food Technology, Islamic University, Kushtia-7003, Bangladesh.

\section{References}

1. Beals KA, Manore MMJJotADA. Nutritional status of female athletes with subclinical eating disorders. 1998, 98:419-425.

2. McArdle WD, Katch FI, Katch VL: Exercise physiology: nutrition, energy, and human performance. Lippincott Williams \& Wilkins; 2010.

3. Burke LM, Kiens B. Ivy JLJJoss: Carbohydrates and fat for training and recovery. 2004, 22:15-30.

4. Croll JK, Neumark-Sztainer D, Story M, Wall M, Perry C, Harnack LJJotADA. Adolescents involved in weight-related and power team sports have better eating patterns and nutrient intakes than non sport-involved adolescents. 2006, 106:709-717.

5. Hornstrom GR, Friesen CA, Ellery JE, Pike KJF, Sciences N: Nutrition knowledge, practices, attitudes, and information sources of mid-american conference college softball players. 2011, 2:109.

6. Dunford M: Sports nutrition: A practice manual for professionals. American Dietetic Associati; 2006.

7. Purcell LKJP. health c: Sport nutrition for young athletes. 2013, 18:200-202.

8. Petrie HJ, Stover EA, Horswill CAJN. Nutritional concerns for the child and adolescent competitor. 2004, 20:620-631.

9. Cupisti A, D'Alessandro C, Castrogiovanni S, Barale A, Morelli EJIJoSN, Metabolism E. Nutrition knowledge and dietary composition in Italian adolescent female athletes and non-athletes. 2002, 12:207-219.

10. Rosenbloom CA, Jonnalagadda SS, Skinner, RJJotAoN. Dietetics: Nutrition knowledge of collegiate athletes in a Division I National Collegiate Athletic Association institution. 2002, 102:418.

11. Cotugna N, Vickery CE, McBee SJTJoSN. Sports nutrition for young athletes. 2005, 21:323-328. 
12. Wong Y, Huang Y-C, Chen S-L, Yamamoto SJNR. Is the college environment adequate for accessing to nutrition education: A study in Taiwan. 1999, 19:1327-1337.

13. Little JC, Perry DR, Volpe SLJJoCH. Effect of nutrition supplement education on nutrition supplement knowledge among high school students from a low-income community. 2002, 27:433-450.

14. Ozdoğan Y, Ozcelik AOJJotISoSN. Evaluation of the nutrition knowledge of sports department students of universities. 2011, 8:11.

15. Zawila LG, Steib C-SM, Hoogenboom BJJoat. The female collegiate cross-country runner: nutritional knowledge and attitudes. 2003, 38:67.

16. Folasire OF, Akomolafe AA, Sanusi RAJGjohs. Does nutrition knowledge and practice of athletes translate to enhanced athletic performance? Cross-sectional study amongst nigerian undergraduate athletes. 2015, 7:215.

17. Beals KA: Nutritional concerns of adolescent athletes. In Nutritional applications in exercise and sport. CRC Press; 2000: 68-89.

18. Webb MC, Beckford SEJJon, metabolism: Nutritional knowledge and attitudes of adolescent swimmers in Trinidad and Tobago. 2014, 2014.

19. Burns RD, Schiller MR, Merrick MA, Wolf KNJJotADA. Intercollegiate student athlete use of nutritional supplements and the role of athletic trainers and dietitians in nutrition counseling. 2004, 104:246249.

20. Rikrak Ch Marak M, Brogen Singh Akoijam P, Luikham. Knowledge of Nutrition and Attitude towards Food Supplements among Sportsperson In Manipur. Journal of Dental Medical Sciences. 2018;17:813.

21. Sedek R, Yih TY. Dietary habits and nutrition knowledge among athletes and non-athletes in National University of Malaysia (UKM). Pakistan Journal of Nutrition. 2014;13:752.

22. Heikkilä $M$, Valve $R$, Lehtovirta $M$, Fogelholm M. Nutrition knowledge among young finnish endurance athletes and their coaches. Int J Sport Nutr Exerc Metab. 2018;28:522-7.

23. Froiland K, Koszewski W, Hingst J. Kopecky LJljosn, metabolism e: Nutritional supplement use among college athletes and their sources of information. 2004, 14:104-120.

24. Torres-McGehee TM, Pritchett KL, Zippel D, Minton DM, Cellamare A. Sibilia MJJoAT: Sports nutrition knowledge among collegiate athletes, coaches, athletic trainers, and strength and conditioning specialists. 2012, 47:205-211.

25. Azizi M, Rahmani-Nia F, Malaee M, Malaee M, Khosravi NJBJoB. A study of nutritional knowledge and attitudes of elite college athletes in Iran. 2010, 4.

26. Davar VJJoHE. Nutritional knowledge and attitudes towards healthy eating of college-going women hockey players. 2012, 37:119-124.

27. Perron M, Endres JJJotADA. Knowledge, attitudes, and dietary practices of female athletes. 1985, 85:573-576.

28. Supriya V, Ramaswami LJIJoIR. Development: Knowledge, attitude and dietary practices of track and field athletic men and women aged 18-22 years. 2013, 2. 
29. Lin W, Yang H-C, Hang C-M, Pan W-HJAPjocn. Nutrition knowledge, attitude, and behavior of Taiwanese elementary school children. 2007, 16:534-546.

30. Lin W: Aspects of nutrition knowledge, attitude, and practice. In Symposium on Nutrition Monitoring and Health Policy Development2004: 3-4.

31. Douglas PD, Douglas JGJJotADA. Nutrition knowledge and food practices of high school athletes. 1984, 84:1198-1202.

32. Foley CS, Vaden AG, Newell GK, Dayton ADJJotADA. Establishing the need for nutrition education: III. Elementary students' nutrition knowledge, attitudes, and practices. 1983.

33. Bazyar H, Zare Javid A, Hosseinpour M, Barati R, Bastampour A, Mousavi F, Dasi E, Haghighizadeh $\mathrm{MH}$, Maghsoumi-Norouzabad L. The relationship between nutritional knowledge and practice with nutrition counseling and education in adult athletes in Ahvaz, 2017. International Journal of Biomedicine Public Health. 2018;1:175-83.

34. Grete RH, Carol AF, Jane EE, Kimberli P: Nutrition knowledge, practices, attitudes, and information sources of mid-american conference college softball players. Food and Nutrition Sciences 2011, 2011.

35. Nazni P, Vimala S. Nutrition knowledge, attitude and practice of college sportsmen. Asian journal of sports medicine. 2010;1:93.

36. BASAMI M, EBRAHIM K, MALAKI A. THE NUTRITIONAL KNOWLEDGE, ATTITUDE AND PRACTICE OF MALE STUDENTS'ATHLETES IN 2014, IR-UNIVERSITY GAMES. 2016.

37. Hornstrom GR: Nutrition knowledge, practice, attitudes, and information sources of Mid-American Conference college softball players. 2007.

\section{Figures}
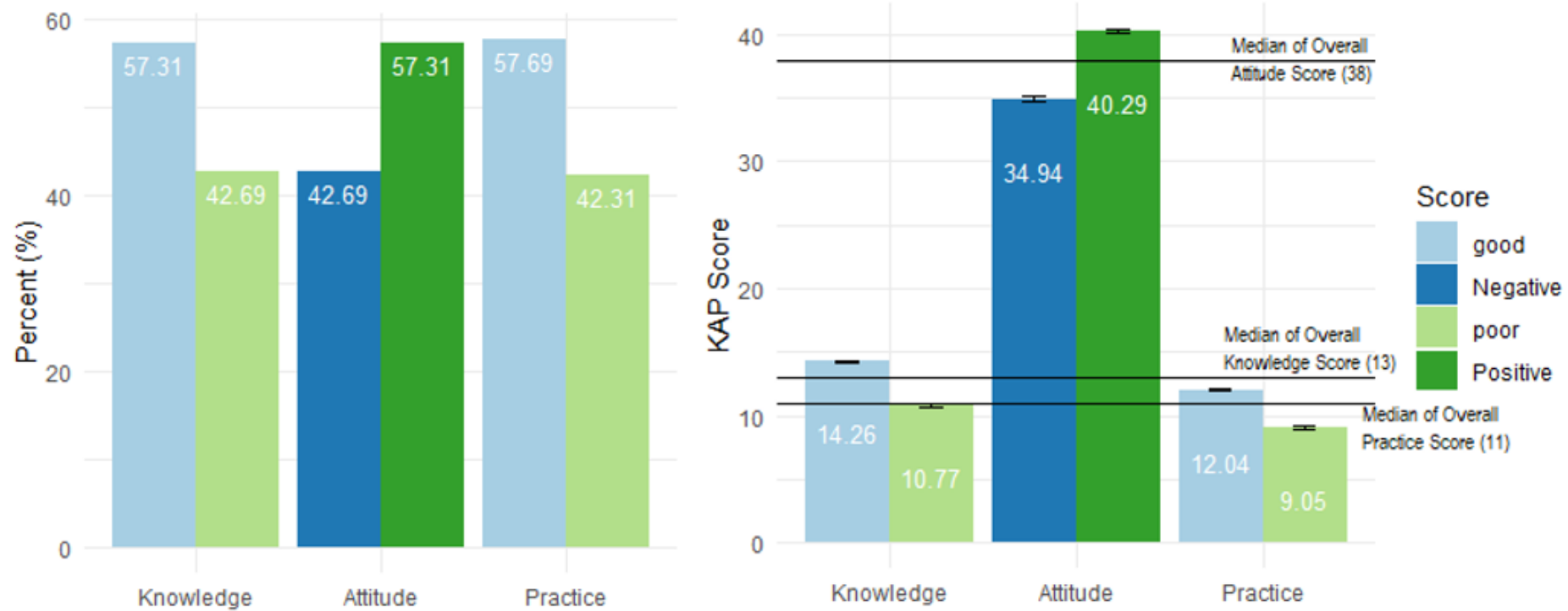

Figure 1 


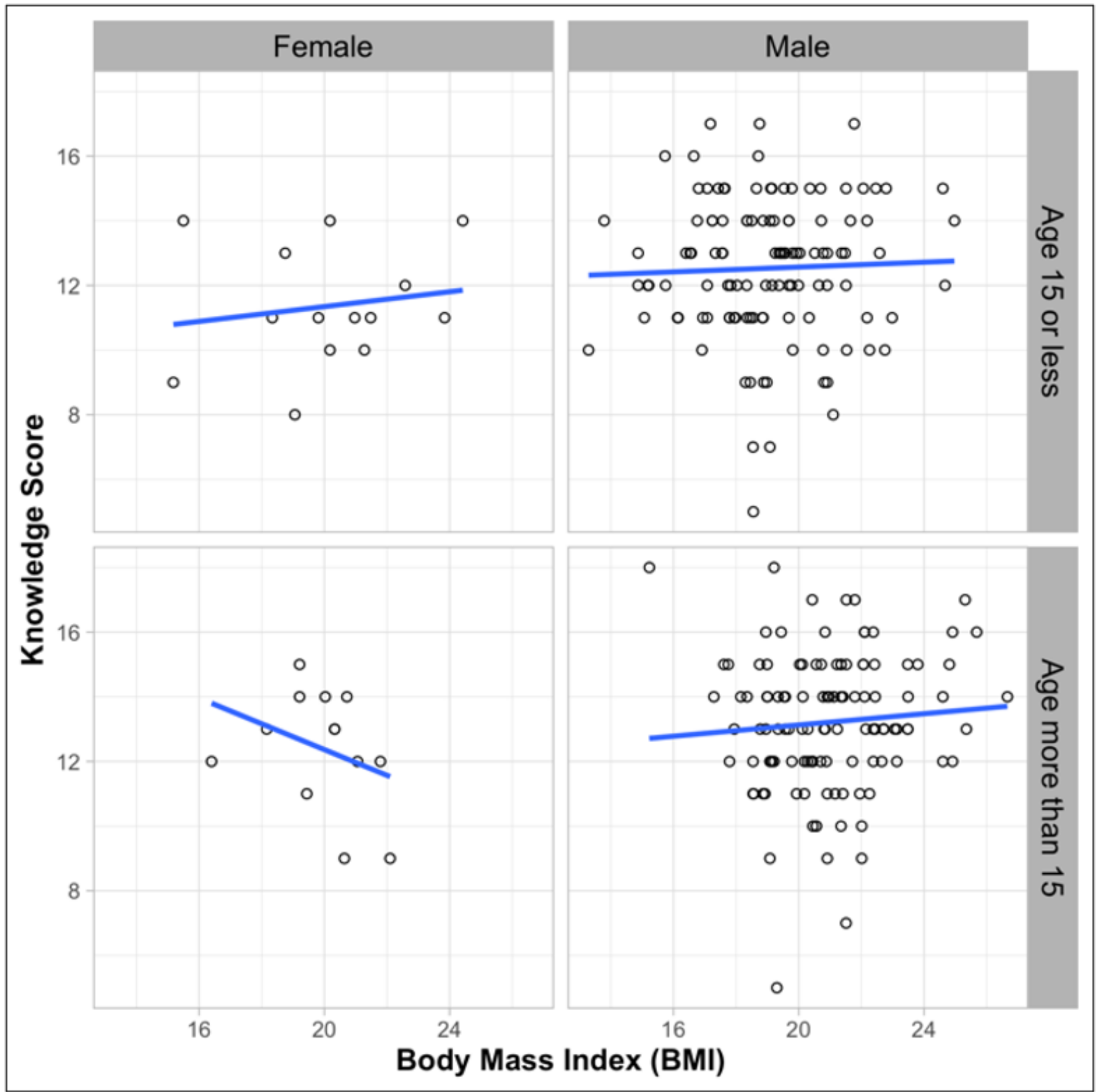

Figure 2

Association of Nutrition Knowledge Score and BMI of Athletes (According to Gender and Age category) 

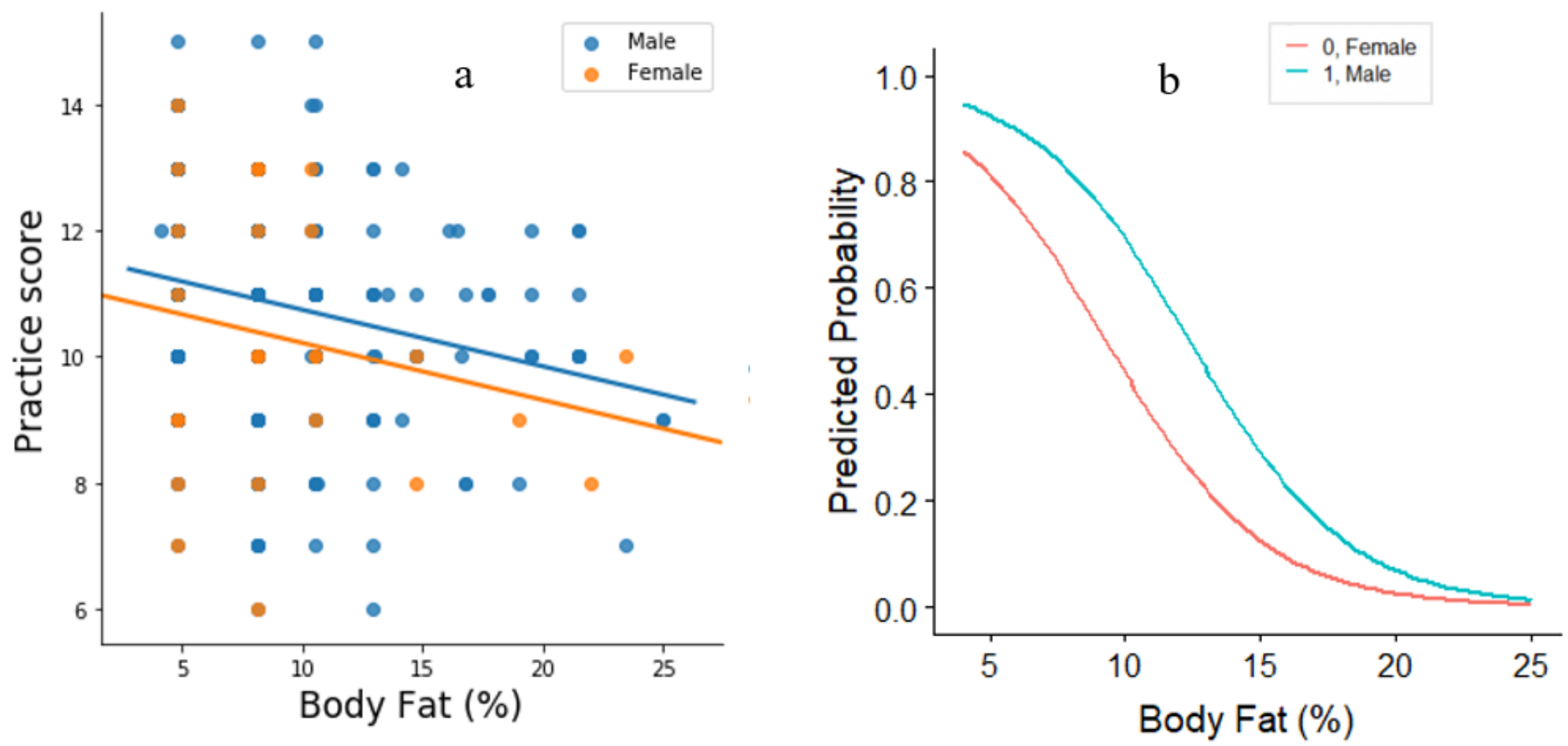

Figure 3

Correlation between practice score and percent body fat of athletes (Picture b) and Predicted Probability of having good practice score against percent body fat. [Both are presented according to gender]
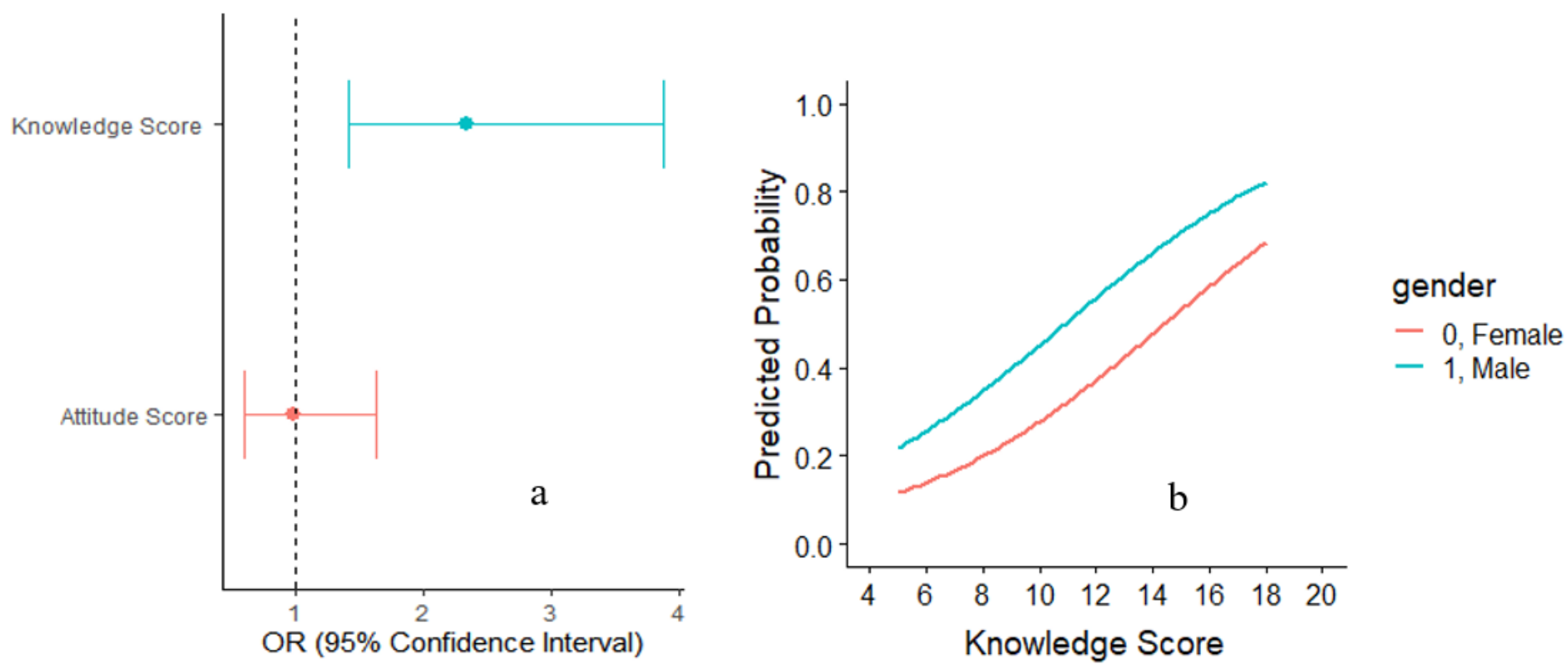

\section{Figure 4}

Adjusted Odd Ratio of the association of practice score with knowledge and attitude score (a) and Predicted Probability of having good practice score against knowledge score. [Logistic model was developed adjusting height (inch), training duration and other variables used in the regression model]. 


\section{Supplementary Files}

This is a list of supplementary files associated with this preprint. Click to download.

- STROBEchecklistv4combined.docx 